\title{
TERRAZAS DE CULTIVO ABANDONADAS EN EL SURESTE PENINSULAR: ASPECTOS EVOLUTIVOS
}

\author{
Juan Antonio Marco Molina \\ Alfredo Morales Gil
}

\section{RESUMEN}

Se realiza una aproximación al tema del abandono de las terrazas de cultivo en el sureste peninsular que, por su particularidad, plantea: problemas conceptuales y de definición del objeto de estudio en el contexto geográfico; el establecimiento de perspectivas y observaciones generales sobre el estado de las terrazas; así como una valoración de los condicionantes evolutivos y las hipótesis de trabajo.

Palabras clave: terrazas de cultivo, abandono, condicionantes de la evolución.

\begin{abstract}
It consists of making an approximation to the subject of abandoned crop terraces in the southeast peninsular which poses conceptual problems and definition of studied object in the geographic context, the establishment of perpectives and general observations about the state of the terraces as well as a valoration of the evolutive conditions and the hypothesis of research.
\end{abstract}

Key words: crop terraces, abandon, conditions in the evolution process.

\section{Introducción}

En el ámbito del sureste peninsular, como en la mayoría de los espacios aledaños, el paisaje agrario adquiere una enorme variedad de formas en las que convergen modos tradicionales y mutaciones relacionadas con las nuevas técnicas. Tanto en un contexto como en el otro, el dinamismo es uno de sus rasgos más llamativos. En el caso que nos ocupa, trataremos sobre parte de un paisaje agrario tradicional, cuya dinámica actual es consecuencia del abandono y desorganización del mismo.

Es éste un tema o línea de investigación que se ha desarrollado en el seno del Instituto Universitario de Geografía de la Universidad de Alicante, de manera que el presente 
trabajo no es la conclusión o resultado final de dicha investigación, sino una reflexión fruto de la experiencia acumulada en infinidad de trabajos de campo, del intercambio de opiniones con miembros del citado Instituto y del enunciado en las líneas de investigación específicas dentro de esta amplia temática. Se trata, por tanto, y, a pesar de presentarse de modo particular, de un trabajo en el que ha influido y colaborado un nutrido colectivo de geógrafos.

El abandono de áreas agrícolas marginales, a consecuencia de las dificultades que ofrecen a su mecanización y a la baja rentabilidad de los cultivos de secano, es un proceso claramente contrapuesto al observado en otras áreas con mejores condiciones para su adaptación a las nuevas técnicas agrarias. La erosión del suelo en el sureste peninsular reviste hoy una potencia jamás alcanzada y su causa primordial ha sido el masivo abandono de terrazgos poco rentables. Terrazas sin cultivo ni repoblación con sus motas u hormas arruinadas, en rápido proceso de abarrancamiento, integran ahora un panorama desolador en grandes áreas de cuencas torrenciales. De esta forma ha desaparecido la doble y benéfica función que ejercían aquéllas de retener suelo y agua, en contrapartida han aumentado las turbideces y coeficientes de escorrentía, acelerándose los procesos de denudación y esqueletización del suelo, consecuencias que se dejan sentir hasta en los llanos de inundación donde la percepción humana popular del fenómeno ha inducido a pensar que, en la actualidad, se ha producido una intensificación de los chubascos, cuando en realidad son mayores las repercusiones negativas de los mecanismos destructivos antes expuestos.

La preocupación de la degradación ambiental por abandono y mal laboreo de las tierras de piedemonte ha estado siempre en las prácticas agrícolas tradicionales que trataban de corregirla. Si bien teóricamente fue expuesta de forma clara por los ilustrados, dentro de un movimiento que propugna una nueva concepción acerca de la naturaleza y su conservación, que es concebida como un sistema y con unos planteamientos conceptuales de gran vigencia actual.

La ordenación de las laderas se concibe con el fin de evitar la degradación ambiental, de buscar una nueva estructuración agraria de los terrazgos y, de esa forma, reducir la erosión en los suelos, así como la defensa de las superficies boscosas en franco retroceso por las diferentes roturaciones de tierras.

A este respecto, destacan dos figuras señeras de la ilustración europea que se preocupan puntualmente por los procesos de degradación ambiental. J. Cavanilles en su obra Observaciones sobre la Historia natural, Geografía, Agricultura, Población y frutos del Reyno de Valencia de 1789 y el italiano G.B. Landeschi en su tratado Saggi di agricultura, (Florencia 1807).

Como referencia inicial del presente siglo destaca la obra de J. Brunhes (1902); posteriormente, son varios los investigadores franceses y españoles preocupados por los procesos de degradación de los aterrazamientos en el Norte de África y en el sureste peninsular, procede recordar los estudios de J. Despois (1949), S. Llobet (1958), J. Vilà (1961), A. Gil Olcina (1967, 1983), M.C. Navarro (1969), A. Morales (1969, 1983, 1989, 1990, 1992, 1993), M. Box (1983, 1986), J.F. Vera y J.A. Marco (1988), J.M. Bernabé (1989) у J.A. Marco (1993).

Se plantean, pues, a continuación, problemas conceptuales, perspectivas y observaciones generales, así como el estado y primeros resultados de algunas hipótesis de trabajo.

\section{Referenciación geográfica del objeto de estudio}

Los sistemas de captación de aguas eventuales para aumentar las disponibilidades hídricas de los cultivos que se practicaban en las sedientas tierras del sureste peninsular 
han sido tradicionalmente dos: el aterrazamiento y compartimentación en pequeñas parcelas de las laderas montañosas y superficies de los glacis, y la derivación mediante presas y canales de las aguas que esporádicamente corrían por los lechos de barrancos y de ramblas —riegos de boqueras - Ambos métodos de acopio de aguas sobre los terrazgos contribuían a laminar las ondas de crecida que se producían después de los chubascos de fuerte intensidad horaria caídos sobre ellos, siempre y cuando éstos no fuesen de proporciones descomunales. De esta forma el hombre, que desde época romana venía roturando estas tierras marginales, intervenía modificando los procesos de escorrentía que de manera natural se debían de producir. Así reducían a algo más de la mitad el volumen de las aguas a evacuar siempre y cuando se tratara de un área que toda ella estuviese en cultivo por este sistema, especialmente si se trataba de glacis y conos de deyección.

Estos mecanismos de aprovechamiento de aguas de lluvia presentaron condiciones de funcionalidad hasta mediados del presente siglo, y desde entonces paulatinamente se han ido desorganizando o han quedado como meras formas residuales, testimonio de un pasado, en las áreas de más precariedad de disponibilidades hídricas, allí donde no fue posible la llegada de aguas alóctonas que favorecieron la expansión de los cultivos de regadío.

En la actualidad, estas técnicas de captación y utilización de aguas esporádicas han sido abandonadas ante la aparición de las nuevas tecnologías agrarias e incluso a veces por la incidencia de la expansión urbana. Efectivamente la introducción de los modernos utillajes de arados arrastrados por potentes tractores de más de $200 \mathrm{CV}$, sustitutos del viejo arado romano con tracción animal, plantearon problemas de maniobrabilidad en el interior de las pequeñas parcelas en el caso de poder llegar hasta ellas, exigiendo por el contrario unos bancales de grandes superficies de en torno a las 8 ó 9 hectáreas frente a las 10 ó 15 áreas de aquéllas. Esta transformación paisajística agraria fue posible sobre las superficies de los glacis, pero no en las laderas montañosas donde las fuertes pendientes lo impidieron. Y así se procedió por gran parte de las tierras de cultivo de secano de las provincias de Alicante, Almería y Murcia durante la década de los sesenta y años sucesivos.

La cuestión relativa a las consecuencias geomorfológicas en tierras abandonadas, requiere o precisa de un planteamiento riguroso desde un principio. No se puede hacer una valoración general de tierras abandonadas, pues no todas son iguales; se hace necesaria una distinción que sirva de contexto referencial, ya que las consideraciones que se realicen serán específicas y subordinadas a ese distingo inicial. Por este motivo el título de la presente comunicación específica claramente que el objeto de estudio son las terrazas de cultivo abandonadas - también merecerían atención las que todavía se trabajan o son de nueva creación-. Y hablamos de terrazas de cultivo porque dicha denominación sirve, a su vez, de referencia sobre la naturaleza del espacio geográfico afectado, y, en especial, de que el aterrazamiento es condigno de la puesta en cultivo de laderas, cotarros o cuestas de pendientes más o menos acusadas.

Sin embargo, esta primera consideración no es del todo cierta, pues, además de laderas sensu lato, las terrazas de cultivo son igualmente frecuentes en vaguadas, incluso cauces, de barrancos y ramblas; son las denominadas «cañadas» o «canyades» en el ámbito alicantino ${ }^{1}$. Son, pues, un elemento más de referencia. Aún más, son otro tipo de terrazas

1 En buena parte de «el campo» del Bajo Segura, la red hidrográfica que hiende la superficie de los glacis, salvo contadas excepciones, se identifica con el topónimo de «cañadas (cauces aterrazados), especialmente en todo el litoral situado al sur de Torrevieja. Igualmente en la vecina Murcia, en el sector del Cabezo la Plata y, mucho más expresivo en este caso, de Cañadas de San Pedro, en un extenso sector constituido por margas yesíferas miocenas entre la continuación oriental de la Cresta del Gallo (al norte) y las sierras Columbares y Altaona (al sur), los topónimos (unidades morfográfico-paisajísticas) más frecuentes son: tollos (cárcavas), cañás (cañadas, cauces aterrazados) y cañizos (cauces menores). 
de cultivo; puesto que, en estos casos, las terrazas ocupan unidades fisiográficas bien definidas cuya dinámica inherente es bien distinta a la de las laderas. Pero no se agotan aquí las posibilidades de distinción; en este último grupo, merecen especial atención no sólo los aterrazamientos para el cultivo, sino también las infraestructuras relacionadas con las técnicas de riego de turbias. Se hablará, por tanto, de terrazas de cultivo en laderas y de terrazas de cultivo en vaguadas o cauces; distingo que es necesario y que ayuda a valorar justamente las consecuencias del abandono en unos y otros casos.

Otra cuestión previa necesitada de precisión es otro de los términos empleados en el enunciado: el abandono; pues, es ambiguo, al tiempo que erróneo. Las tierras de cultivo, en nuestro caso, las terrazas, dejan de cultivarse, pero a menudo soportan otro tipo de aprovechamiento, especialmente el pastoreo; y, por otras parte, es preciso evaluar la duración de dicho «abandono» o cambio de uso, no sólo por relacionar dicha magnitud temporal en los procesos observados, sino también para tomar referencias precisas sobre los episodios meteorológicos que han podido influir en estos espacios.

Es, pues, obligado tener como referencias, por un lado los aprovechamientos posteriores al abandono del cultivo y, por otro, la frecuencia con que se han producido episodios torrenciales desde que las terrazas en cuestión han dejado de cultivarse. En definitiva, se trata de referenciar la evolución de estas tierras con parámetros reales, y no mediante extrapolaciones, proyecciones de futuro o simulaciones.

\section{Fenómenos observados}

Para conseguir dichos objetivos es preciso reconocer, casi inventariar, cuáles son los procesos erosivos y fenómenos que afectan a estos sectores; así como señalar el significado de cada uno de ellos, en un contexto específico y en unas determinadas circunstancias. Por ello, conviene introducir los distingos señalados, e incluso, indicar los matices que convengan y, así, dentro de las terrazas de cultivo en laderas, comenzamos por detallar que en las laderas constituidas por margas son frecuentes las pequeñas cárcavas, erosión en surcos, así como fenómenos de erosión en túnel (pipes). Pero también se ha comprobado que estos procesos suelen ir precedidos por desmoronamiento de los muretes de piedra seca, y que no siempre desembocan en fenómenos de erosión acelerada que arpen el conjunto de la ladera. En muchas ocasiones, como sucede en la mayor parte de los valles afluentes del río Amadorio - fosas tectónicas-, en las terrazas de cultivo abandonadas, el desmoronamiento del «marge» (murete de piedra) se produce a base de «trencs» sucesivos, también conocidos como «solsides» (pequeños desmoronamientos-deslizamientos), que lo van eliminando; de manera que la repetición de este fenómeno termina por provocar la sustitución de un escalonamiento brusco como el que definen las terrazas, por una sucesión de rellanos separados por taludes.

Otro tanto se puede afirmar de las laderas aterrazadas sobre glacis o derrubios de ladera, como las situadas al sur y sureste del Cabeçó d‘'Or, donde un comportamiento idéntico de desmoronamiento del «marge» provoca la acumulación del material del murete al pie de la terraza, y propicia, por la pedregosidad, que gran parte de las partículas finas que caen a continuación permanezcan más o menos trabadas o retenidas en el talud de bloques y cantos, de tal modo que se va produciendo una regularización del desnivel existente entre dos terrazas contiguas: talud que es colonizado relativamente rápido, en especial por el Brachypodium retusum; en definitiva, lo que se observa en muchos sectores es, pues, un restablecimiento del perfil de la ladera de forma más o menos acusada.

Sin embargo, dicha situación final sólo se ha observado en aquellos sectores en los que la edad del abandono se remonta a la mitad del siglo actual, a los años sesenta, o incluso, 

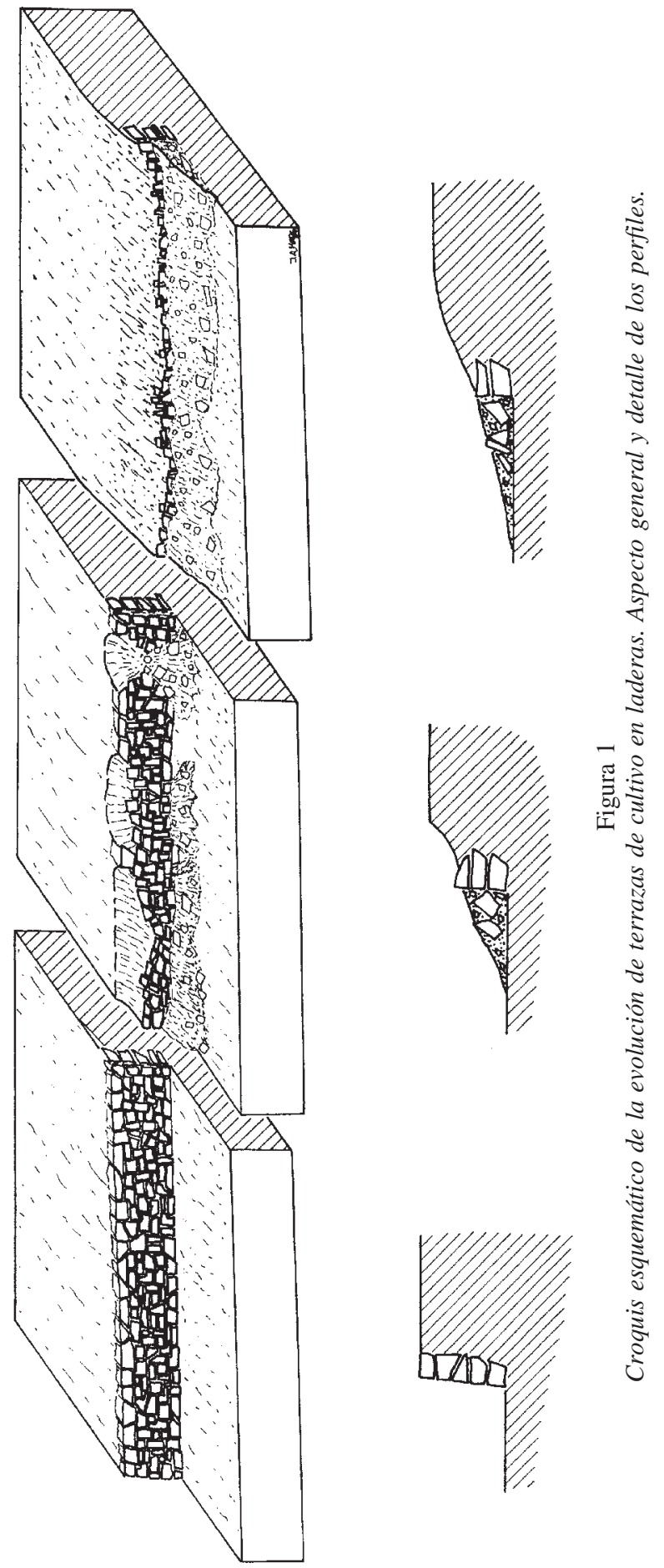
a abandonos acaecidos durante el primer cuarto de siglo. Lo más habitual, no obstante, son situaciones intermedias que, como todo lo inacabado, lo incompleto, se perciben como estados regresivos o negativos, cuando, en realidad, se está produciendo un reajuste del equilibrio de las laderas en cuestión; proceso en el que intervienen energías que en unos momentos actúan de forma contrapuesta, pero que, en definitiva, convergen en el mismo sentido. Hasta que ello sucede, cualquiera de estas laderas podría ejemplarizarse como un lugar en el que se han desencadenado serios procesos de erosión acelerada.

Tanto en un contexto como en el otro, es decir, tanto sobre margas como en depósitos detríticos cuaternarios, las consecuencias del abandono no se limitan a las manifestaciones señaladas, que destacan más por lo evidente de las mismas, que por la importancia de su significado, tal y como se ha indicado en un sentido evolutivo. No debemos olvidar que estas terrazas, al igual que las instaladas en cauces y vaguadas, son, mientras se practican las labores agrícolas, un complejo absorbente artificial debido a la textura que adquiere el suelo con el labrado; esta particular forma de incrementar la infiltración (uno de los principales objetivos de las terrazas), se desvanece de forma progresiva a medida que transcurre el tiempo desde que se abandonan dichas prácticas, pues tanto las litofacies como las formaciones superficiales ganan en compacidad y disminuye su capacidad de infiltración. Es, pues, evidente, que todo ello redunda en un aumento de la escorrentía, de los flujos vehiculados por los cauces; consecuencias que ya han sido señaladas como factores determinantes en la génesis de las crecidas (MORALES, BRU y BOX, 1983: GIL et al., 1983; MORALES y BOX, 1986; GIL et al., 1986); y por tanto, que adquieren verdadera dimensión geográfica.

En cuanto a las terrazas de cultivo en cauces, tanto si se trata de litofacies margosas como si son los materiales detríticos cuaternarios, los fenómenos observados coinciden plenamente con los señalados en primer lugar para el otro tipo de terrazas, es decir, variadas formas de vaciado, si bien, lo más habitual es que, tras procesos de entubamiento o erosión en túnel (piping), se evoluciones hacia ramblizos (gullies). Se trata pues de unas consecuencias totalmente previsibles en estas unidades morfográficas: la reapertura y excavación de los cauces que habían sido ordenados y puestos en cultivo.

Pero en estas unidades, las intervenciones humanas no se han limitado a la construcción de terrazas, sino que también se ha acometido la realización de verdaderas obras hidráulicas: presas de derivación, de sangración o azudes. En todas ellas, si el objetivo último era el de control y manejo del agua, además, como obstáculos transversales o trazados en los cauces, también desempeñaban un importante papel como elemento de retención de los débitos sólidos. Su construcción determinó el aluvionamiento y colmatación de considerables tramos de barrancos y ramblas en espesores que, en ocasiones, crecían a medida que lo hacían estas obras hidráulicas. Entradas en desuso y abandonadas, el deterioro y fatal destrucción de las mismas, implica la movilización del relleno que colmataba los cauces; se produce así, una recuperación de la fisonomía de estas unidades que son los cauces, con las evidentes consecuencias geomorfológicas que implica, en conjunto, la desorganización de un paisaje agrario tradicional. Es, por tanto, en estos espacios, donde la dinámica morfológica alcanza las cotas más elevadas de ablación de material; aunque, eso sí, sin olvidar que éste es resultado directo de una actuación humana, se podría decir que se produce la erosión de un relleno antropogénico.

\section{Valoración de los condicionantes de la evolución}

Para cubrir este apartado, vamos a partir de la base de que existe gran variedad de situaciones morfológicas en las terrazas de cultivo abandonadas — estadios o fases evolu- 
tivas-, y, dicha diversidad responde a las interacciones existentes entre un cúmulo de factores que han condicionado la evolución morfológica de las mismas. De ellos se diferencia entre los básicos o características propias de las terrazas de cultivo (naturaleza del soporte) y los dinámicos o condicionantes de la evolución propiamente dichos (motores de la misma).

Entre los primeros, destacar, en primer lugar, y por razones ya expuestas, la unidad morfológica aterrazada, pues de ello deriva la naturaleza de las litofacies y formaciones superficiales, así como la pendiente; y, en segundo lugar, la arquitectura de las terrazas, ya que en ocasiones, resulta decisiva en la evolución de las mismas la mayor o menos consistencia de la fábrica de los muretes; en este sentido, sería precisa una rigurosa clasificación de tipos.

Por otra parte, los condicionantes dinámicos considerados son:

- abióticos, además de la componente lítica ya indicada, las condiciones climáticas y, especialmente, el calendario de episodios torrenciales;

-bióticos, tanto al considerar la fauna como posible desencadenante de ciertos procesos, como a la vegetación, elemento de estabilización de las laderas;

$-\mathrm{y}$, por último, los antropogénicos, aprovechamientos posteriores al cultivo y duración del abandono.

Que la evolución de las terrazas de cultivo abandonadas es resultado de la combinación de todos los elementos o factores señalados, es un hecho que no ofrece dudas. No obstante, la diversidad de situaciones se relaciona con el mayor o menor protagonismo de unos y otros; de tal modo que, a continuación, se valoran algunos ejemplos del estado de las terrazas y su relación con los elementos que se presentan como más decisivos; es, en realidad, un modo de presentar algunas hipótesis de trabajo.

El fenómeno que adquiere mayor relevancia, desde un punto de vista geomorfológico, es el de la erosión de los materiales que constituyen las terrazas. Es indudable que en la génesis de estos procesos resulta esencial el concurso de precipitaciones de elevada intensidad y lluvias torrenciales; pero su eficacia depende, igualmente, de las condiciones de escorrentía, pues en el caso de las terrazas, en general, la efectividad de estas aversiones meteorológicas es inversamente proporcional a la duración del abandono, de manera que si son coetáneos éste y la génesis de uno de estos episodios, el arramblamiento, erosión y degradación de la arquitectura de las terrazas, son mayores. De ahí que, para comprender y explicar las discontinuidades espaciales que se observan en el estado de las terrazas sea preciso considerar, de forma específica, todos estos parámetros; sólo así se puede comprender la realidad y no llegar a especulaciones de futuros inciertos, especialmente cuando los mecanismos implicados se caracterizan por una gran discontinuidad en su remanencia.

Así se pudo comprobar en las terrazas de cultivo en ladera situadas en el paraje de Pichoc (San Vicente del Raspeig), donde, sobre derrubios de ladera, la «oportunidad» de un episodio torrencial en otoño de 1991, cuando estas terrazas se habían abandonado el año anterior, propició la aparición y desarrollo de unos galaches («garras del diablo»), surcos que se hundían entre 10 y $15 \mathrm{~cm}$ (aproximadamente hasta donde llegaba el arado) en los materiales que constituyen la terraza; situación que podría indicar procesos de erosión acelerada, pero que, en realidad, al no reproducirse el fenómeno meteorológico, se han paralizado y han sido sustituidos, hasta el momento, por un proceso de regeneración natural de la vegetación que se encuentra en su estadio inicial: han aparecido las primeras especies — pioneras - que han iniciado el cubrimiento del suelo, concretamente, este papel lo desempeñan Helichrysum stoechas y, en menor medida, Thymelaea hirsuta: esta es la realidad. 
En este caso que se acaba de detallar, se ha destacado el papel de un elemento biótico como contrapunto a la erosión, la regeneración de la vegetación. Sin embargo, otro elemento biótico, la fauna, a menudo desempeña el papel contrario, el de desencadenante de ciertos procesos erosivos. Durante los trabajos de campo, resulta esencial la encuesta y, en repetidas ocasiones, la opinión de los agricultores coincide en señalar que la abundante presencia de conejos degrada la arquitectura de las terrazas, pues sus intrincadas madrigueras, son origen de su ruptura. Planteamiento aplicado a las terrazas cultivadas, pero igualmente válido para las terrazas de cultivo abandonadas.

En efecto, en la partida conocida como la Lloma Badá (Petrer), así como en los frentes de cuesta miocenos del Campo de Cartagena (Casas de Truyols, Tercia, Sucina, Corvera,...), se ha podido observar que en terrazas de cultivo abandonadas en ladera margosa, junto a los procesos de regeneración natural de la vegetación (prácticamente se podría hablar de un romeral, en el primero de los casos; mientras que en otros ámbitos se ha reconstituido un auténtico espartizal) y la degradación de la arquitectura de las terrazas, que ha deparado la práctica desaparición de los muretes y la formación de un talud, la actividad biológica de los logomorfos citados, ha sido tan intensa que las parcelas, tanto en el frente como en la superficie, están recorridas por galerías y perforadas por oquedades. A la vista de esta situación, no extraña que, en el mismo sector, la circulación de las aguas de escorrentía por dichos conductos y su salida por el frente de las terrazas, haya podido propiciar el vaciado parcial de las mismas que se manifiesta en pequeños ramblizos y colapsos, similares a los procesos de entubamiento.

En relación con el factor antropogénico, dos han sido los aspectos señalados: la duración o edad de abandono y la existencia de un aprovechamiento con posterioridad al cultivo.

Con respecto al primero, tanto en terrazas de cultivo en laderas como en cauces y vaguadas aterrazadas, se observa una relación muy directa: a mayor duración del abandono, proporcional regeneración de la vegetación y mayor aproximación a la morfografía primitiva de la unidad morfológica en cuestión.

En cambio, cuando lo que se produce es un cambio de aprovechamiento, generalmente dedicación a pastos, a la vez que se produce un estancamiento o paralización de la evolución vegetal, se mantienen durante más tiempo las condiciones morfológicas propias de los sectores recientemente abandonados y, por tanto, expuestos a procesos erosivos «acelerados»; no sólo por la desaparición de la cobertera vegetal, sino más bien por el poder destructivo de las pezuñas de ovejas y cabras que son capaces, incluso, de romper los muretes de piedra seca.

Como ejemplos que se ajustan a las consideraciones hechas, citar que en los piedemontes y glacis del Medio y Alto Vinalopó, Altiplano de Jumilla-Yecla, Sierras Occidentales Murcianas, Cuenca de Mula, Campo de Lorca, Sierra de los Filabres, Valle del Almanzo$\mathrm{ra}, . .$. , allí donde se han dejado de cultivar las terrazas en laderas de fuerte pendiente, los ganados que transitan por ellas van provocando el desplome de los muros de contención, acelerando la pérdida de suelo. En los casos del Alto Vinalopó y Altiplano de JumillaYecla, donde se ha procedido al abandono subvencionado del cultivo de viñas, al no ser sustituido por otro, dadas las características de semiaridez de las tierras, se han convertido en inmensos pastizales; así en las zonas de convergencia de glacis, se ha producido el desmoronamiento de las pequeñas pedrizas de acumulación antrópica (fruto del despedregado de las parcelas) que separaban parcelas de grandes dimensiones. La remoción de muretes y un pastoreo intenso han conducido a estas terrazas a no tener la más mínima protección vegetal y, en años de acusada indigencia pluviométrica, se pueden localizar los rebaños a larga distancia por las enormes polvaredas que levantan. Del mismo modo, sirva 

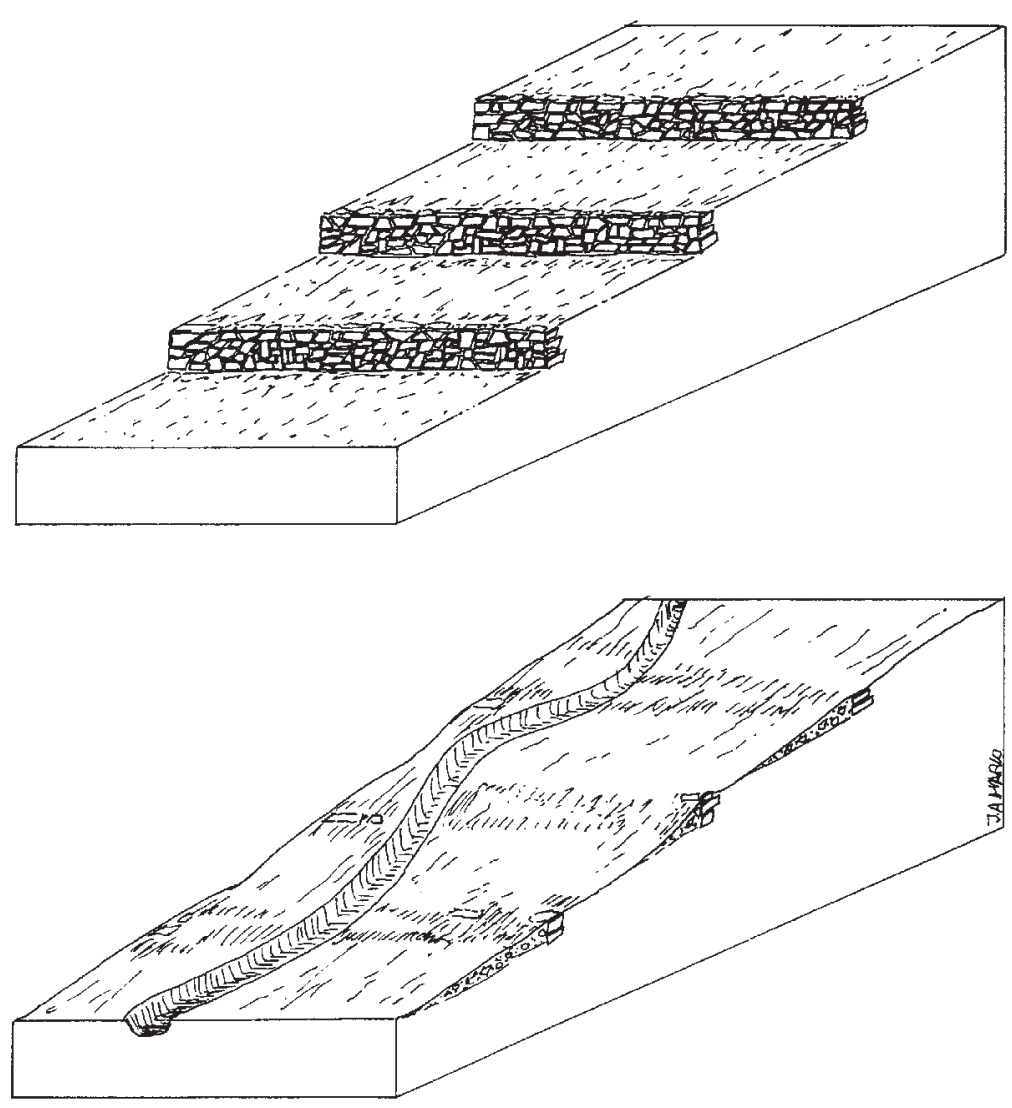

Figura 2

Croquis esquemático del aspecto primitivo y estado actual de las terrazas de cultivo en cauce (basado en el ejemplo del barranco de la Serra de Santa Pola, Alicante).

como ejemplo el del cauce aterrazado de un barranco encajado en la vertiente septentrional de la Serra de Santa Pola: a lo largo del cauce, prácticamente desde su cabecera, se suceden las terrazas que, adaptándose a la fisonomía de aquél, son cada vez de mayores dimensiones, menores pendientes y menos pedregosas; muy posiblemente, estos rasgos justifican, en gran medida, el ritmo de abandono de las mismas. De manera que son las más altas las primeras en dejar de ser cultivadas, mientras que en las más bajas, además de ser más tardío el abandono, más bien se ha producido un cambio de aprovechamiento, e incluso en la actualidad, se dedican a pastos.

Desde un punto de vista biogeográfico, las consecuencias son bien contrastadas, pues, si en las situadas aguas arriba se ha producido una importante recuperación de la cubierta vegetal (cubrimiento próximo al 100\%), compuesta por un lentiscar-espinar (Pistacia lentiscus y Rhamnus lycioides son las especies dominantes); por contra en las más bajas, la evolución de la vegetación está detenida en las primeras fases: junto a especies herbáceas (pasto), sólo destaca la presencia de aquéllas que, en este ámbito, tienen una clara vocación de plantas pioneras como la bufalaga (Thymelaea hirsuta).

En el plano geomorfológico se marca nuevamente el contraste entre los dos ámbitos 
señalados. En las terrazas más bajas, donde se desarrolla el pastoreo y, donde además, las pendientes son menores, se conserva bastante bien la arquitectura de las terrazas, sólo se ha observado el deterioro de la parte superior de algunos muretes y la aparición de «un golpe de cuchara» o «trenc» en una de las terrazas que salva un desnivel mayor (sucedió en octubre de 1982). Por contra, en las terrazas más altas, al igual que la reconstitución de la cubierta vegetal está muy avanzada — próxima al óptimo-, morfológicamente también se advierte una profunda transformación hacia lo que podríamos denominar óptimo morfológico. Los desniveles que primitivamente definían las terrazas, han desaparecido casi por completo, sólo se manifiestan aquellos en los que la fábrica de los muretes es más robusta y sólida, y en las terrazas más próximas al tramo bajo, donde el abandono ya es algo posterior; al tiempo que, frente al fondo completamente plano condigno del aterrazamiento, actualmente se marca un estrecho cauce que, por otra parte, está colonizado por la comunidad vegetal señalada con anterioridad.

Se robora, de este modo, que la evolución de las terrazas es resultado de un proceso muy complejo cuya comprensión obliga a manejar multitud de elementos y que, en definitiva, hemos tratado de jerarquizarlos, si bien, también se advierte que dicha jerarquización ni puede, ni debe plantearse como un esquema rígido.

\section{Referencias bibliográficas}

BRUNHES, J. (1902): L'irrigation dans la Peninsule Iberique et dans l'Afrique du Nord. París.

CAPOT-REY, R. (1961): «Les paysages ruraux des steppes et des deserts», Géographie Générale, A. Jornaux (dir.), Gallimard, 1.883 pp., París.

CAVANILLES, A. J. (1797): Observaciones sobre la Historia natural, Geografía, Agricultura, Población y frutos del Reyno de Valencia, Tomo II, Imprenta Real, Madrid.

DESPOIS, J. (1949): «L'utilisation des eaux de crue dans les pays arides de l'Afrique du Nord. Exemple des plaines du Hodna». Congreso Internacional de Geografía de Lisboa, 4 vols.

GIL OLCINA, A. (1967): El campo de Lorca. Tesis doctoral, 771 folios, Valencia.

GIL OLCINA, A. et al. (1983): Lluvias torrenciales e inundaciones en Alicante. Inst. Universitario de Geografía. Universidad de Alicante.

GIL OLCINA, A. et al. (1986): Inundaciones en la ciudad y término de Alicante. Univ. AlicanteAyto. de Alicante.

LANDESCHI, G.B. (1807): Saggi di agricultura, Florencia, 1770.

LLOBET, S. (1958): «Utilización del agua en la región semiárida de Huércal-Overa (Almería)». Estudios Geográficos, no 70, pp. 5-21.

MARCO MOLINA, J.A. (1993): «Rocas blandas y riesgos en el sur de la Comunidad Valenciana (España)». Investigaciones Geográficas, n 11, pp. 237-249, Alicante.

MORALES GIL, A. (1969): «El agua con aguas de avenidas en las laderas subáridas». Papeles del Departamento de Geografía de Murcia, I.

- (1989): «Abandono y desorganización de los sistemas tradicionales de riego de turbias. Su incidencia en la escorrentía». Los paisajes del agua, Universidades de Valencia y Alicante.

MORALES GIL, A., BRU RONDA, C. y BOX AMORÓS, M. (1983): «Las avenidas en los barrancos de las Ovejas y del Agua Amarga. Alicante, octubre de 1982». Estudios Geográficos, $\mathrm{n}^{\circ} 170-171$, Madrid.

MORALES GIL, A. y BOX AMORÓS, M. (1986): «El aprovechamiento del agua y los suelos en un dominio semiárido: la cuenca del barranco Blanco. Agost (Alicante)». Investigaciones Geográficas, $\mathrm{n}^{\circ}$ 4, Inst. Universitario de Geografía, Univ. de Alicante.

NAVARRO NAVARRO, Mº C. (1969): «Problemas agrarios en un sector de clima semiárido: el campo de Águilas» Revista de Geografía, vol. III, n 1, pp. 5-39, Barcelona.

VERA REBOLLO, J.F. y MARCO MOLINA, J.A. (1988): «Impacto de los usos del suelo y erosión en cuencas vertientes del sur del País Valenciano». Investigaciones Geográficas, $\mathrm{n}^{\circ}$ 6, Inst. Universitario de Geografía, Univ. de Alicante. 